\title{
Impact of pulmonary vein ostia anatomy on efficacy of cryoballoon ablation for atrial fibrillation
}

\author{
Jakub Baran ${ }^{1, A-D, F}$, Roman Piotrowski ${ }^{1, B}$, Agnieszka Sikorska ${ }^{1, B}$, Ilona Kowalik², ${ }^{2,}$ Tomasz Kryński ${ }^{1, D}$,
} Stec Sebastian ${ }^{3, \mathrm{E}}$, Piotr Kułakowski ${ }^{1, \mathrm{C}-\mathrm{F}}$

A - Research concept and design, B - Collection and/or assembly of data, C - Data analysis and interpretation,

D - Writing the article, E - Critical revision of the article, F - Final approval of article

1 Postgraduate Medical School Warsaw, Szpital Grochowski

2 2nd Department of Coronary Artery Disease, Institute of Cardiology, Warsaw, Poland

3 Chair of Electroradiology, Faculty of Medicine, University of Rzeszow, Rzeszow, Poland

Address for correspondence:

Jakub Baran, Postgraduate Medical School Warsaw, Szpital Grochowski

email: j.baran@sampi.pl

Roman Piotrowski, Postgraduate Medical School Warsaw, Szpital Grochowski

email: rpiotrow@op.pl

Agnieszka Sikorska, Postgraduate Medical School Warsaw, Szpital Grochowski

email: agnsikorska@wp.pl

Ilona Kowalik, 2nd Department of Coronary Artery Disease, Institute of Cardiology, Warsaw, Poland

email: ilonamich@wp.pl

Tomasz Kryński, Postgraduate Medical School Warsaw, Szpital Grochowski

email: tomasz.krynski@gmail.com

Stec Sebastian, Chair of Electroradiology, Faculty of Medicine, University of Rzeszow, Rzeszow, Poland

email: smstec@wp.pl

Piotr Kułakowski, Postgraduate Medical School Warsaw, Szpital Grochowski

email: kulak@kkcmkp.pl

Received: 28.10 .2016

Revised: 29.12.2016

Accepted: 29.12.2016

\section{Key words:}

\section{atrial fibrillation, cryoballoon ablation, pulmonary vein ostium.}

\section{Introduction}

Cryoballoon (CB) ablation has become a widely accepted method for treatment of patients with atrial fibrillation (AF) and pulmonary vein isolation (PVI) ${ }^{[1]}$. Due to the fact that the size and shape of the balloon are predefined, PV anatomy, including number of veins, presence of common trunk,

\section{Methods Patients}

The study group consisted of 32 consecutive patients (21 males, mean age $56 \pm 12$ years) who underwent $C B$ ablation in our institution in 2012 and 2013 and who had computed tomography (CT) of the left atrium (LA) and PV performed not longer than 48 hours before ablation. Demographic and clinical characteristics are presented in Table 1. The data were collected prospectively; however, the analysis was of a retrospective nature. angulation, ostium area and ovality, may have an impact on CB ablation efficacy. A few studies which addressed this issue provided inconsistent results ${ }^{[2-6]}$. Therefore, the aim of our study was to examine whether PV ostia anatomy is related to the acute and long-term effects of $\mathrm{CB}$ of $\mathrm{AF}$.

\section{Pre-procedural imaging}

One or two days before the procedure a contrast-enhanced CT scan with segmentation of the LA and PV was performed using a 16-slice scanner (Aquilion 16 CT Scanner, Toshiba, Otawara, Japan). Scanning was performed at $135 \mathrm{kVp}$ and an effective tube current of $500 \mathrm{mAs}$. The contrast enhancement was obtained by using a total of $80-100 \mathrm{ml}$ of contrast agent (Ultravist 370, Bayer, Berlin, Germany) injected at a flow rate of $4.5 \mathrm{ml} / \mathrm{s}$ and followed by a 30-ml saline flush. The recon- 
structed images were transferred to a CT workstation (3Dnet Suite, Biotronics3D, London). Reconstructions were performed along the long axis of the V1 segments of the pulmonary veins as well as 3D surface reconstructions of the left atrium and the pulmonary veins with manual measurements of the ostial diameters.

The PV ostium area and ovality index were measured according to Schmidt et al. ${ }^{[6]}$. After CT segmentation, the PV were measured at the ostial level. The ovality index, which expresses the degree of deviation from perfect circularity, was calculated according to the formula: $2 x(a-b) /(a+b)$ where " $a$ " is the longest diameter, and " $b$ " is the shortest diameter. The higher the index of ovality, the more oval (less circular) the PV ostium is. The ostial area was measured manually by delineation of the PV ostium directly on the CT platform and the numerical result in $\mathrm{mm} 2$ was provided automatically by the system.

Representative examples of the measurements of PV ostia area and ovality are presented in Figure 1.

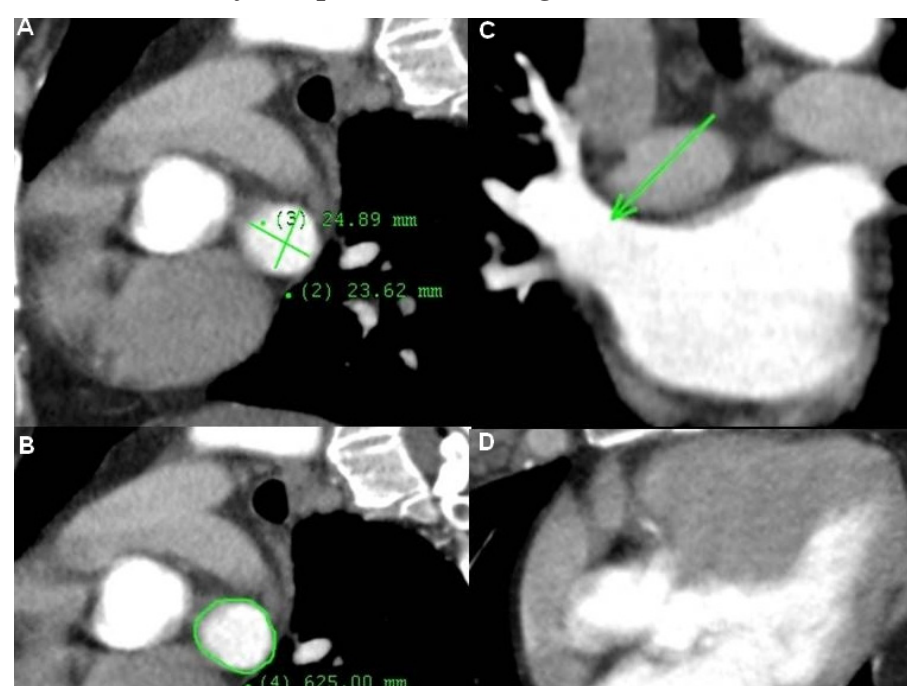

Figure 1 Representative examples of measurement of horizontal and vertical diameter of the right superior pulmonary vein $(\mathrm{A})$ and area measurement of the vein ostium (B). In panels (C) and (D) right superior pulmonary vein in horizontal and vertical plane is displayed.

\section{Ablation}

Cryoballoon AF ablation was performed in patients in conscious sedation according to the standard scheme. After transseptal puncture a $28 \mathrm{~mm}$ CB (Arctic Front or Arctic Front Advance, Medtronic, US) was introduced into the LA using a steerable sheath (FlexCath Advance, Medtronic, US). We usually used two applications per vein lasting $240 \mathrm{~s}$, trying to engage different branches of the PV. If after two freezes PVI was not achieved, an additional one or two freezes with different $\mathrm{CB}$ angulation and cannulating other PV branches were performed. If PVI was achieved promptly (within $90 \mathrm{~s}$ from the start of $\mathrm{CB}$ ablation) shortening of duration of application to $180 \mathrm{~s}$ and the need for a second application were left to the discretion of the operator. PV occlusion was confirmed using injection of radiographic contrast. We usually first isolated the left superior PV (LSPV), followed by the left inferior PV (LIPV), right superior PV (RSPV) and right inferior PV (RIPV).
During ablation of the RSPV and RIPV, pacing from the right subclavian vein was performed in order to avoid phrenic nerve palsy. PVI was assessed using a circular Achieve catheter (Medtronic, US), which was introduced inside the PV before each application and remained until PVI was achieved and application finished. The LabPro Bard electrophysiology system (Bard, US) was used for intracardiac and ECG recordings.

The presence of hypertension, congestive heart failure, coronary artery disease, diabetes, and chronic renal failure was identified according to standard definitions.

\section{Follow-up}

The patients were followed in the outpatient clinic, where they underwent 24-hour Holter ECG monitoring 3, 6 and 12 months after the procedure. Between these visits, the patients attended primary care units. Patients were encouraged to record ECG in case of palpitation or other symptoms suggesting cardiac arrhythmia and also to examine their pulse on the radial or carotid artery. The ablation was regarded as unsuccessful when the patients had symptomatic AF recurrences confirmed on ECG or AF episodes lasting $>30 \mathrm{~s}$ recorded during Holter ECG monitoring, symptomatic or not. The use of antiarrhythmic drugs after ablation was left to the discretion of primary care physicians, although both patients and their doctors were encouraged to stop antiarrhythmic therapy after a 3-month blanking period if there was no AF recurrence.

\section{Statistical analysis}

The results are presented as mean \pm standard deviation or numbers and percentages. Differences between quantitative variables were assessed using the two-tailed Student t-test and differences between qualitative parameters were assessed using the chi square test with or without Yates' correction, or Fisher's exact test, where appropriate. ANOVA with post-hoc Tukey test was used for multiple comparisons. A p value < 0.05 was considered significant.

\section{Results}

The study group consisted almost exclusively (all but one) of patients with paroxysmal AF. The most common concomitant disease was hypertension (66\%). The mean HAS-BLED and CHA2DS2-VASc scores were low $(0.91 \pm 0.64$ and $1.34 \pm 1.19$, respectively). Only 5 (16\%) patients had a HASBLED score of 2 and only $4(13 \%)$ had a CHA2DS2Vasc score $\geq 3$ (Table 1 ).

In 32 patients $128 \mathrm{PV}$ were identified. Two patients had a left common trunk. No additional PV was present.

The mean values of ostial area and ovality as well as nadir temperature and cumulative duration of cryoapplications are presented in Table 2 with corresponding $p$ values for comparisons between the veins shown in Table 3. The RSPV ostium was the largest, and that of LIPV was the smallest. The most oval vein was the LSPV, followed by the LIPV and RSPV. Left veins had more oval (less circular) ostia than the right veins. The lowest temperature was achieved in the RSPV and the least negative in the RIPV. Differences between temperature achieved in the RSPV and in both inferior PV were signifi- 
Table 1. Demographic and clinical characteristics of the studied patients.

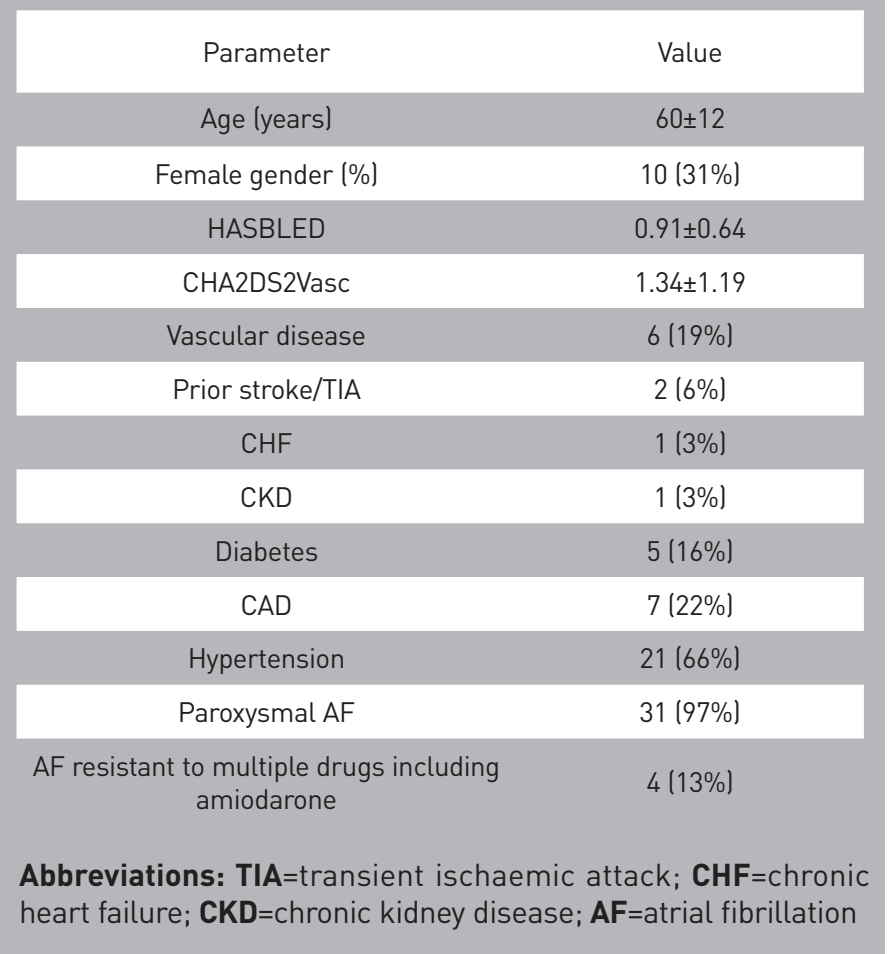

cant. The longest cumulative application time was needed to isolate the LSPV, followed by the LIPV, RIPV and RSPV. The differences in application time between the left and right PV were significant.

During the procedure, 122 out of 128 veins were isolated (95\%). In two patients one vein and in another two patients two veins were not isolated. All $128 \mathrm{LSPV}$ were isolated, whereas in one LIPV, two RSPV and three RIPV complete isolation was not achieved.

The follow-up duration was $33 \pm 8$ months. During this period, $22(69 \%)$ patients remained free from AF recurrences. The remaining 10 patients had AF recurrences and eight of them underwent repeated ablation procedures with a good outcome, whereas the two remaining patients preferred to continue pharmacological therapy. In the group without AF recurrences, only one patient had a non-isolated vein (RIPV), whereas in 10 patients with AF recurrences, 3 had non-isolated PV.
The comparison between effectively and non-effectively treated groups is presented in Table 3. Out of PV anatomical parameters patients with AF recurrences had significantly greater ostial area in both inferior PV compared with patients without $\mathrm{AF}$ recurrences. The mean values of the ovality index did not significantly differ between effectively and non-effectively treated patients. Also the mean values of nadir negative temperature and total duration of application were similar in both groups. Also complete PVI was associated with a good outcome $(\mathrm{p}=0.044)$.

Out of demographic and clinical variables, non-effectively treated patients tended to be more often of female gender and had a history of AF resistant to multiple antiarrhythmic agents, including amiodarone (all differences not significant).

\section{Discussion}

Cryoballoon ablation has become a standard approach for invasive treatment of patients with $\mathrm{AF}$ in recent years. It has been shown that the efficacy of this technique is similar to the standard RF-based AF ablation and may be associated with lower hospital readmissions, direct electrical cardioversions and repeated ablations ${ }^{[1,7]}$. Also the learning curve is much shorter than in the case of RF ablation, which gives a promise for more widespread use of this technique than difficult pointby-point RF ablation ${ }^{[8,9]}$.

Cryoballoon-based AF ablation is a technique which is highly dependent on PV anatomy. The size and shape of a $\mathrm{CB}$ is constant, whereas the anatomy of a PV is variable. Such parameters as PV ostia area and ovality as well as the PV angulation with respect to the left atrial wall and the angle between the septum and ostia of the right PV may have a potentially important impact on balloon proper closure of PV ostia, PVI achievement and clinical outcome.

A few studies have already addressed this issue. Sorgente et al. ${ }^{[5]}$ reported that vein ostium ovality and orientation predicted the degree of PV occlusion, especially in the left-sided PV. However, that study did not provide any data on efficacy of the procedure, and the ovality index was calculated using a different formula than that used in our study, which may account for the discrepant results of our study and that of Sorgente et al. Also Schmidt et al. ${ }^{[6]}$ suggested that the ovality index predicted complete occlusion and AF recurrences after ablation. Their study differed from ours in having more patients included in the analysis and closer follow-up (7-day Holter ECGs).

Table 2. Mean values of individual pulmonary vein ostial area and ovality as well as nadir temperature and cumulative duration of application.

$\begin{array}{ccccc}\text { Vein } & \text { Ostium area }(\mathrm{mm} 2) & \text { Ovality index } & \text { Temperature }\left({ }^{\circ} \mathrm{C}\right) & \text { Duration of application }(\mathrm{s}) \\ \text { LSPV } & 227.1 \pm 73.1 & 0.2617 \pm 0.3509 & -47.1 \pm 10.9 & 565 \pm 209.7 \\ \text { LIPV } & 197.1 \pm 47.6 & 0.2446 \pm 0.3677 & -45.9 \pm 8.6 & -50.7 \pm 6.0 \\ \text { RSPV } & 294 \pm 136.8 & 0.1804 \pm 0.3210 & -45.6 \pm 6.5 & 393.6 \pm 159.3\end{array}$


Table 3. Mean values of individual pulmonary vein ostial area and ovality as well as nadir temperature and cumulative duration of application.

\begin{tabular}{|c|c|c|c|c|}
\hline & Ostium area & Ovality index & Temperature & Duration \\
\hline LSPV vs LIPV & 0.06961 & 0.85218 & 0.63744 & 0.21704 \\
\hline LSPV vs RSPV & 0.02358 & 0.351739 & 0.11996 & 0.00058 \\
\hline LSPV vs RIPV & 0.07251 & 0.06399 & 0.52977 & 0.00311 \\
\hline LIPV vs RSPV & 0.00065 & 0.46659 & 0.01748 & 0.02389 \\
\hline LIPV vs RIPV & 0.00150 & 0.11602 & 0.88682 & 0.07135 \\
\hline RSPV vs RIPV & 0.51648 & 0.41458 & 0.00358 & 0.75812 \\
\hline
\end{tabular}

Our study showed that out of anatomical parameters of AF ablation than PV ostium ovality. When the PV ostium is PV ostia, the ostium area may play a greater role for CB-based bigger than the CB diameter, it may be difficult to isolate the

Table 4. Comparison of demographic, clinical and pulmonary vein anatomical parameters between patients without ( $\mathrm{n}=22$ ) and with $(n=10)$ recurrences of AF after cryoballoon ablation.

\begin{tabular}{|c|c|c|c|}
\hline Parameter & Effective $n=22$ & Not effective $n=10$ & $P$ value \\
\hline Age (years) & $56.5 \pm 13.2$ & $54.8 \pm 8.4$ & 0.72 \\
\hline Female gender (\%) & $5(23 \%)$ & $5(50 \%)$ & 0.12 \\
\hline HASBLED & $0.95 \pm 0.56$ & $0.8 \pm 0.75$ & 0.53 \\
\hline CHA2DS2Vasc & $1.4 \pm 1.11$ & $1.2 \pm 1.3$ & 0.65 \\
\hline Diabetes & $3(14 \%)$ & $2(20 \%)$ & 0.65 \\
\hline CAD & $6(27 \%)$ & $1(10 \%)$ & 0.27 \\
\hline Hypertension & $15(68 \%)$ & $6(60 \%)$ & 0.65 \\
\hline AF resistant to amiodarone & $2(9 \%)$ & $2(20 \%)$ & 0.38 \\
\hline ALL 4 veins isolated & $21(95 \%)$ & $7(70 \%)$ & 0.044 \\
\hline $\begin{array}{c}\text { PV ostium area }(\mathrm{mm} 2) \\
\text { LSPV } \\
\text { LIPV } \\
\text { RSPV } \\
\text { RIPV }\end{array}$ & $\begin{array}{c}215.3 \pm 69.5 \\
185.2 \pm 37.1 \\
263.2 \pm 110.5 \\
231.1 \pm 57.8\end{array}$ & $\begin{array}{c}254.7 \pm 73.9 \\
225.6 \pm 57.6 \\
365.8 \pm 162.7 \\
369.2 \pm 144.4\end{array}$ & $\begin{array}{l}0.188 \\
0.038 \\
0.063 \\
0.001\end{array}$ \\
\hline $\begin{array}{l}\text { PV ostium ovality index } \\
\text { LSPV } \\
\text { LIPV } \\
\text { RSPV } \\
\text { RIPV }\end{array}$ & $\begin{array}{c}0.2613 \pm 0.38 \\
0.2316 \pm 0.4 \\
0.1724 \pm 0.35 \\
0.0882 \pm 0.2\end{array}$ & $\begin{array}{l}0.2625 \pm 0.28 \\
0.2731 \pm 0.27 \\
0.1980 \pm 0.25 \\
0.2037 \pm 0.17\end{array}$ & $\begin{array}{l}0.99 \\
0.77 \\
0.84 \\
0.14\end{array}$ \\
\hline $\begin{array}{c}\text { Nadir temperature }\left({ }^{\circ} \mathrm{C}\right) \\
\text { LSPV } \\
\text { LIPV } \\
\text { RSPV } \\
\text { RIPV }\end{array}$ & $\begin{array}{l}-49.8 \pm 6.3 \\
-44.5 \pm 8.1 \\
-50.5 \pm 4.2 \\
-46.4 \pm 6.9\end{array}$ & $\begin{array}{l}-46.3 \pm 7.5 \\
-48.8 \pm 8.9 \\
-51.2 \pm 8.8 \\
-44.0 \pm 5.0\end{array}$ & $\begin{array}{l}0.2 \\
0.23 \\
0.73 \\
0.38\end{array}$ \\
\hline $\begin{array}{l}\text { Cumulative duration of applicat } \\
\qquad \begin{array}{c}\text { (s) } \\
\text { LSPV } \\
\text { LIPV } \\
\text { RSPV } \\
\text { RIPV }\end{array}\end{array}$ & $\begin{array}{c}549.2 \pm 141.3 \\
502 \pm 152.7 \\
419.4 \pm 145.3 \\
464.8 \pm 172.3\end{array}$ & $\begin{array}{c}600 \pm 308.3 \\
495 \pm 249.1 \\
336.8 \pm 173.4 \\
364.4 \pm 130\end{array}$ & $\begin{array}{l}0.54 \\
0.93 \\
0.14 \\
0.18\end{array}$ \\
\hline
\end{tabular}


vein at the level of the PV ostium. Isolation may be achieved by freezing more deeply, inside the vein; however, this should be avoided because it carries the risk of causing PV stenosis or phrenic nerve palsy. Thus, a greater number of applications and repositioning of a balloon may be needed, and because the closure of the PV ostium is not complete, a less negative temperature may be achieved.

Our study did not show that the degree of ovality of PV ostia is associated with the outcome, whereas in two other studies such a relationship was found ${ }^{[5,6]}$. Although it seems logical that the more oval (less circular) the PV, the more difficult it is to obtain complete closure of the vessel with a non-compliant circular balloon, it does not have to be necessarily true. The PV ostia are usually quite compliant, and by pushing the balloon slightly harder towards the vein, a good contact is achieved without introducing the device too distally into the vein. Thus, ovality of the PV per se may not be as important as the magnitude of PV ostium area.

The nadir negative temperature and cumulative duration of application of cryoenergy were not associated with the outcome in our study. The former parameter has been shown by some authors to predict ablation efficacy ${ }^{[10]}$; however, when duration of cryoapplication and, consequently, temperatures achieved are modulated by the speed of producing PVI (rapid PVI often results in shorter cryoapplications) and by safety measures (exceeding $-55-60^{\circ} \mathrm{C}$ is not advisable), these parameters may play a smaller role in predicting ablation efficacy. Lack of a relationship between cumulative duration of cryoenergy and ablation efficacy may be explained by the fact that longer applications might mean that the cryoenergy was applied to more "difficult" veins, thus not necessarily producing complete PV occlusion and PVI.

There are other anatomical issues relevant to the efficacy of CB-based AF ablation such as vein angulation or the presence of a common trunk. Some authors have reported that angulation may affect proper closure of the PV ostium by balloon ${ }^{[5]}$; however, we did not examine this parameter in our study. The presence of a common trunk, usually on the left side, is regarded by some as an obstacle to obtain proper proximal $\mathrm{PVI}^{[3,4]}$ whereas others did not find such a relationship ${ }^{[2]}$. Our finding that the area of the RSPV is greater than that of other $\mathrm{PV}$ is in line with the results of Guler et al. ${ }^{[3]}$, who demonstrated that greater RSPV size was associated with more AF recurrences after ablation. In our study we had two patients with a left common trunk, and in both patients CB ablation was successful.

Out of demographic and clinical variables, female gender and history of AF resistant to amiodarone tended to be associated with poorer outcome; however, these differences did not achieve statistical significance, most likely due to the small number of patients. Complete PVI was associated with good outcome, which confirms the well-known fact that isolation of all four PV is necessary to prevent AF recurrences.

Limitations. Our study has several limitations. The study group was small, and more patients may be required to draw definite conclusions. Secondly, we did not analyse PV angulation, the degree of occlusion, the time to PVI or the thawing time, which are regarded as important parameters for predicting CB efficacy. Thirdly, during the follow-up period we used only 24-hour ECG Holter recordings and not longer-term continuous ECG monitoring. Thus, some asymptomatic AF episodes might have been overlooked, although the length of the follow-up in our study was quite long, approaching 3 years.

Conclusions: Ablation efficacy for atrial fibrillation using cryoballoon may be related to pulmonary vein ostium area. This finding together with other anatomical parameters reported in the literature may suggest that pre-procedural imaging may be helpful in deciding which technique - cryoballoon (28 $\mathrm{mm}$ or $23 \mathrm{~mm}$ ) or RF - is more suitable for a given patient.

\section{References}

1. Kuck K-H, Brugada J, Fürnkranz A, et al. Cryoballoon or radiofrequency ablation for paroxysmal atrial fibrillation. N Engl J Med. 2016;374(23):2235-45.

2. Khoueiry Z, Albenque J-P, Providencia R, et al. Outcomes after cryoablation vs. radiofrequency in patients with paroxysmal atrial fibrillation: impact of pulmonary veins anatomy. Eur Eur Pacing Arrhythm Card Electrophysiol J Work Groups Card Pacing Arrhythm Card Cell Electrophysiol Eur Soc Cardiol. 2016;18(9):1343-51.

3. Güler E, Güler GB, Demir GG, et al. Effect of pulmonary vein anatomy and pulmonary vein diameters on outcome of cryoballoon catheter ablation for atrial fibrillation. Pacing Clin Electrophysiol PACE. 2015;38(8):989-96.

4. Kubala M, Hermida J-S, Nadji G, Quenum S, Traulle $\mathrm{S}$, Jarry G. Normal pulmonary veins anatomy is associated with better AF-free survival after cryoablation as compared to atypical anatomy with common left pulmonary vein. Pacing Clin Electrophysiol PACE. 2011;34(7):837-43.

5. Sorgente A, Chierchia GB, de Asmundis C, et al. Pulmonary vein ostium shape and orientation as possible predictors of occlusion in patients with drug-refractory paroxysmal atrial fibrillation undergoing cryoballoon ablation. Eur Eur Pacing Arrhythm Card Electrophysiol J Work Groups Card Pacing Arrhythm Card Cell Electrophysiol Eur Soc Cardiol. 2011;13(2):205-12.

6. Schmidt M, Dorwarth U, Straube F, et al. Cryoballoon in AF ablation: impact of PV ovality on AF recurrence. Int J Cardiol. 2013;167(1):114-20.

7. Kuck K-H, Fürnkranz A, Chun KRJ, Metzner A, et al. Cryoballoon or radiofrequency ablation for symptomatic paroxysmal atrial fibrillation: reintervention, rehospitalization, and quality-of-life outcomes in the FIRE AND ICE trial. Eur Heart J. 2016; 37: 2858-2865

8. Calkins H, Kuck KH, Cappato R, et al. 2012 HRS/ EHRA/ECAS Expert Consensus Statement on Catheter and Surgical Ablation of Atrial Fibrillation: recommendations for patient selection, procedural techniques, patient management and follow-up, definitions, endpoints, and research trial design. Eur Eur Pacing Arrhythm Card Electrophysiol J Work Groups Card 
Pacing Arrhythm Card Cell Electrophysiol Eur Soc Cardiol. 2012;14(4):528-606.

9. Providencia R, Defaye P, Lambiase PD, et al. Results from a multicentre comparison of cryoballoon vs. radiofrequency ablation for paroxysmal atrial fibrillation: is cryoablation more reproducible? Eur Eur Pacing Arrhythm Card Electrophysiol J Work Groups Card Pacing Arrhythm Card Cell Electrophysiol Eur Soc Cardiol. 2016 euw080. [Epub ahead of print]

10. Siklódy $\mathrm{CH}$, Minners J, Allgeier M, Allgeier H-J, et al. Pressure-guided cryoballoon isolation of the pulmonary veins for the treatment of paroxysmal atrial fibrillation. J Cardiovasc Electrophysiol. 2010;21(2):120-5. 